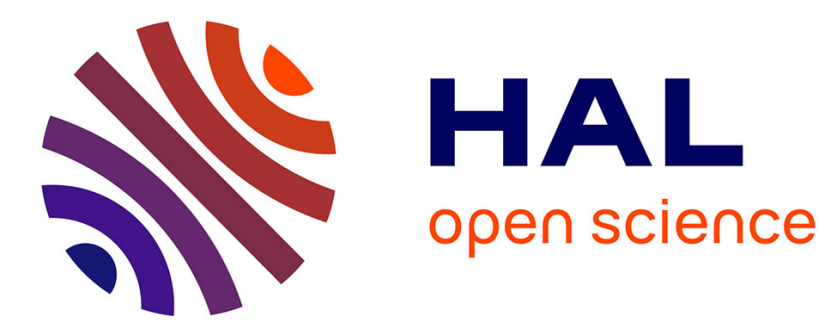

\title{
CFD modeling of buoyancy driven cavities with internal heat source-Application to heated rooms
}

\author{
C. Teodosiu, Frédéric Kuznik, R. Teodosiu
}

\section{To cite this version:}

C. Teodosiu, Frédéric Kuznik, R. Teodosiu. CFD modeling of buoyancy driven cavities with internal heat source-Application to heated rooms. Energy and Buildings, 2014, 68, pp.403-411. 10.1016/j.enbuild.2013.09.041 . hal-01003063

\section{HAL Id: hal-01003063 \\ https://hal.science/hal-01003063}

Submitted on 9 Jun 2014

HAL is a multi-disciplinary open access archive for the deposit and dissemination of scientific research documents, whether they are published or not. The documents may come from teaching and research institutions in France or abroad, or from public or private research centers.
L'archive ouverte pluridisciplinaire HAL, est destinée au dépôt et à la diffusion de documents scientifiques de niveau recherche, publiés ou non, émanant des établissements d'enseignement et de recherche français ou étrangers, des laboratoires publics ou privés. 


\title{
CFD modeling of buoyancy driven cavities with internal heat source -
}

\section{Application to heated rooms}

\author{
C. Teodosiu ${ }^{1}$, F. Kuznik ${ }^{2,3}$, R. Teodosiu ${ }^{1}$ \\ ${ }^{1}$ Faculty of Building Services and Equipment, Technical University of Civil Engineering, \\ Bucharest 020396, Romania \\ ${ }^{2}$ Université de Lyon, 69361 Lyon Cedex 07, France \\ ${ }^{3}$ INSA-Lyon, CETHIL UMR5008, 69621 Villeurbanne Cedex, France \\ Corresponding author. Tel.: +40 2125246 20; Fax: +40 2125268 80; \\ Email: cteodosiu@yahoo.com
}

\begin{abstract}
The aim of this work is to examine the capacity and the accuracy of a CFD (Computational Fluid Dynamics) model to characterize the thermo-aeraulic behavior of a heated room. Firstly, we present a brief description of the experimental set-up taken into account. Afterwards, we focus on the main features of our numerical model (that strongly influence the accuracy of results): computational domain geometry and discretization, turbulence model, near wall treatment, radiation model and thermal boundary conditions. In addition, we present here a simplified approach in order to integrate a pure buoyancy source within our model, based on a volumetric heat generation rate (taking into account only the source power and its dimensions) which is uniformly distributed within the heater. Furthermore, detailed experimental - numerical comparisons are given with regard to heat transfer to the walls as well as to heat source behavior and plume characteristics. The results obtained demonstrate that the CFD method employed in this work leads to reliable results. Consequently, this approach can be useful in detailed studies dealing with thermal comfort, indoor air quality and energy consumption for heated rooms. Finally, the simplified method presented here, concerning the integration of the heat source in the CFD model, can be effortlessly extended for other localized heat sources that differ in power, heat emission mode (convection or radiation), shape or size.
\end{abstract}

Keywords: CFD - Computational Fluid Dynamics modeling; buoyancy driven cavity; heat source; thermal plume. 


\section{Introduction}

Thermal coupling "enclosure-heat source" is of particular importance in the field of building physics. An accurate representation of this phenomenon allows us to correctly predict the induced flows inside the room. This leads also to good estimations of thermal comfort as well as indoor air quality. On the other hand, an appropriate description of the thermal behaviour of a heated enclosure helps to accurately assess the heat transfer phenomena that take place across the building envelope. Consequently, the energy consumption will be exactly evaluated. Furthermore, such studies allow optimising the design of heating systems.

Therefore, many studies have been devoted in recent times to these issues concerning the coupling between rooms and their heating systems. However, we note that most of these analyses are based on zonal models. In brief, their theory is based on dividing the enclosure air volume in several air zones and employing heat and mass balance equations for each of these zones (each zone is supposed to be perfectly mixed with uniform temperature or other scalars, e.g. moisture, chemical species, pollutant concentration, etc.). The zone partition is frequently accomplished using experimental data for the main directions of the air flow, depending on the configuration taken into account (ventilated room, heated room, etc.). An exhaustive review of zonal modelling, from the pioneering work till recent developments is given in [1,2]. Nevertheless, it is worthwhile to mention that the zone models are not appropriate for studies dealing with nonuniform zone air temperatures, including convective heating [3].

Instead, the CFD technique has been successfully employed in various fields. We cite here only several recent applications related to modeling of technical systems in the field of building equipment (the topic of our study): ventilation system for hospital operating room [4], effect of inlet position ("under-floor" air distribution versus "over-head" air distribution) for an air conditioned office room [5], natural ventilation of buildings [6], radiation panel cooling system installed in walls [7], radiant cooling ceiling [8], radiant tube heater [9], evaporator and condenser of a rooftop air-conditioning unit [10], under-floor heating system [11], skirting boards heating system [12]. It is interesting to note that the CFD approach has been also used for peculiar studies, e.g. improving building design and thermal comfort for a 100-year-old Buddhist temple [13]. 
Consequently, in order to study buoyancy driven cavities with internal heat source (heated rooms), our choice is based on CFD modeling. This approach is able to provide more complete and pertinent results than the zonal models. In addition, the computation time is more than reasonable due to hardware amazing evolution nowadays.

In this context, the main objective of this work is to methodically assess the ability and the accuracy of a CFD model to study a heated room. The precision of such a numerical simulation is clarified by comparing the results achieved with those from experiments. Therefore we first present briefly the experimental set-up followed by the principal characteristics of our numerical model.

\section{Experimental set-up}

Our study is entirely based on the experimental results presented in [14]. This experimental program has been selected as we have all the information needed for the numerical model and its validation (especially concerning the boundary conditions and the dynamic and thermal plume fields). It is also worthwhile to mention that the laboratory arrangements and the testing methods of this experimental set-up have been used to determine the standard thermal output of the heating appliances fed with water supplied by a remote heat source.

The experimental analyses were performed in a climatic test room $\left(4 \times 4 \times 2.8 \mathrm{~m}^{3}\right)$ in steady state conditions. All the walls of this full scale test cell are in contact with adjacent controlled temperature areas (see Figure 1). Due to precise experimental methods [14], the thermal conductivity of the test cell walls (marine plywood) has been determined: $0.108 \pm 0.002 \mathrm{~W} / \mathrm{m}^{\circ} \mathrm{C}$. The emissivity of the walls inner surface has been also evaluated: $0.95 \pm 0.05$ [14]. These data allow us to well characterize the thermal state of the room envelope in our numerical model.

The measurements carried out in [14] can be classified in boundary conditions and field data. Concerning the first group of measurements, it is mainly constituted by the surface temperatures of the walls. The sensors used are type $\mathrm{T}$ (copper-constantan) thermocouples, distributed on both sides of the enclosure walls (accuracy $\pm 0.25^{\circ} \mathrm{C}$ ). In addition, there were platinum resistance thermometers - RTD sensors Pt $100 \Omega$ (accuracy $\pm 0.1^{\circ} \mathrm{C}$ ) to control the air temperature within the thermal guards of the test cell (Figure 1). Finally, probes of the same type were used to determine the surface temperature of the heat emitters. 


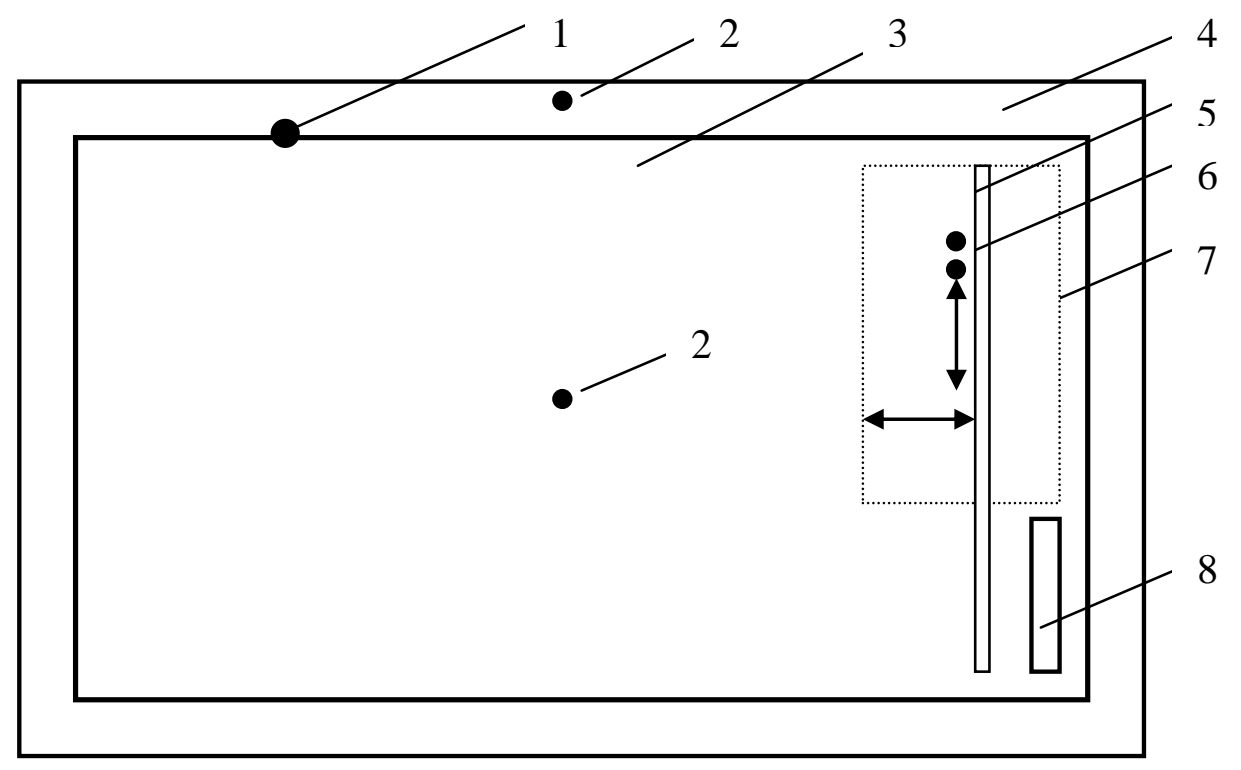

Figure 1. Experimental set-up scheme [14]

1. thermocouple (walls temperature); 2. temperature air sensors; 3. test room

4. adjacent thermal guard; 5 . device used to move the sensors; 6 . velocity and temperature plume sensors; 7. examined plume area; 8. radiator.

Dynamic and thermal fields within the volume of the experimental cell were acquired in the median plane of the cell by means of a "self-travelling" device remotely controlled. The sensors mounted on the experimental device are the following:

- air velocity, omnidirectional hot-film transducers

- air temperature, thermocouples type $\mathrm{T}$ in the plume region and platinum resistance thermometers in the other volume of the enclosure

Figure 2 shows the mesh adopted for the measurements points in the plume above the heat source.

We take into consideration in these work two experimental tests on the same radiator but with changed boundary conditions. The radiator is a steel single panel heater (connected at a hot water heating system) and its dimensions are $1.2 \times 0.013 \times 0.7 \mathrm{~m}^{3}$ (1098 W standard thermal output). Table 1 shows the experimental conditions during the two configurations taken into account. The test room walls identification is based on the assumption that the vertical wall adjacent to the radiator is the south wall. 


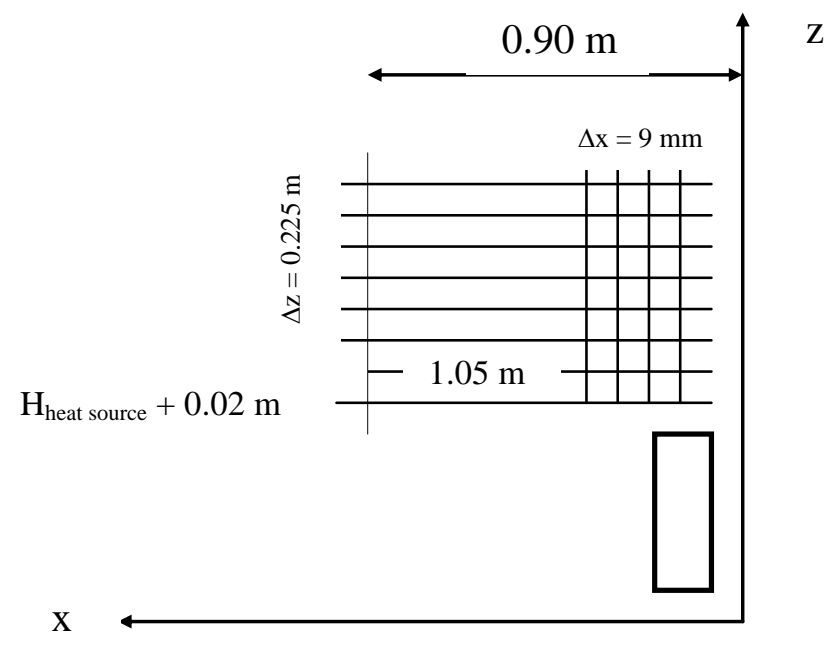

Figure 2. Mesh of the measurements points in the plume [14]

We notice that in the case of the test R1, the vertical wall adjacent to the radiator (south) and the opposite one (north) correspond to external cold ambient conditions. On the contrary, the south wall and the ceiling represent exterior conditions in the test $\mathrm{R} 2$.

Table 1. Experimental conditions [14]

\begin{tabular}{cccccccc}
\hline \multirow{2}{*}{ Test } & \multirow{2}{*}{ Heater power $(\mathrm{W})$} & \multicolumn{5}{c}{ External adjacent thermal guard temperature $\left({ }^{\circ} \mathrm{C}\right)$} \\
& & South & North & East & West & Ceiling & Floor \\
\hline R1 & 900 & 13.4 & 14.4 & 20.0 & 20.0 & 19.8 & 19.8 \\
R2 & 881 & 13.6 & 20.0 & 20.0 & 20.0 & 14.6 & 20.0 \\
\hline
\end{tabular}

\section{Numerical model}

Before proceeding with the presentation of the numerical model, it is worthwhile to emphasise the characteristics of the considered tests.

In general, it is convenient to use dimensionless number in order to describe physical phenomena. In the case of natural convection, the characteristic dimensionless number is the Rayleigh number, defined by the following expression:

$\mathrm{Ra}=\frac{\mathrm{g} \beta \Delta \mathrm{TL}^{3}}{\mathrm{a} v}$

where $\mathrm{L}$ is the characteristic length and $\Delta \mathrm{T}$ is the reference temperature difference. These quantities are accompanied by the fluid properties (in this case air): $\beta$ - thermal expansion 
coefficient; $v$ - kinematic viscosity; a - thermal diffusivity. Finally, g is the acceleration due to gravity.

This number is used to classify the three flow regimes typically encountered: steady laminar flow, transition flow (unsteady laminar) and turbulent flow. In accordance with the results presented in [15], based on extensive literature review (both experimental and numerical studies) dealing with the classic problem of airflow within square buoyancy driven cavities (known as "window problem"), the value of the Rayleigh number corresponding to the laminar-turbulent transition is $2.1 \div 2.2 \times 10^{6}$ for perfectly conducting walls and $1.7 \div 2.25 \times 10^{8}$ for adiabatic conditions. In addition, a comprehensive study of buoyancy-driven convective interaction phenomena in enclosures can be found in [16].

On the other hand, it must be taken into consideration the fact that our geometrical and thermal conditions are distinct and very distant from the "window problem" configuration. Consequently, it is difficult to define the two reference parameters (length and temperature difference) in order to assess the value of the Rayleigh number in our case. In general, the height of the cavity is considered as characteristic length, while the reference difference temperature is based on the maximum difference temperature between two opposite vertical walls. Unfortunately, in our situation, we can only employ the first hypothesis concerning the reference length, the choice of the enclosure height being logical. On the contrary, for the temperature difference, we can not use the opposite vertical temperature difference since for the test R1 (Table 1) these two walls are cooled. Nevertheless, for instance, based on the temperature difference between "North" wall (considered warm wall) and "South" all (considered cooled wall) in the case of the test R2, we determine a value of $4.5 \times 10^{6}$ for the Rayleigh number. This value, compared to the results of "window problem", show that the airflow within the enclosure in our case is well situated in a turbulent flow regime.

Moreover, it must be added that the airflow within the cavity is primarily generated by the heat source and secondary by the walls boundary layers. This is the reason why we do not correlate thereafter our results with data based on classical "window problem" configurations.

It is also interesting to mention that despite the fact that the literature is poor in numerical studies on our topic, the conclusions of the few existing works show that in order to improve the predictions of CFD modeling for buoyancy driven enclosures with internal heat sources (including heated rooms), it is recommended to revise or enhance the following issues: 
discretization, turbulence modeling, airflow near wall treatment, radiation modeling, thermal boundary conditions and heat source description. As a result, we focus further on these factors that present a major impact on the computations. All these issues have been assembled on the basis of a general-purpose, finite-volume, Navier-Stokes solver (Fluent version 6.1).

\subsection{Computational domain geometry and discretization}

Concerning the geometry, this is identical to the configuration of the experimental study (Figure 3). The heat source (steel panel heater) is represented by its real dimensions (including its thickness). Given the results obtained (see the section on the heat source integration within the model), this approach provides excellent numerical description of the heat emitter.

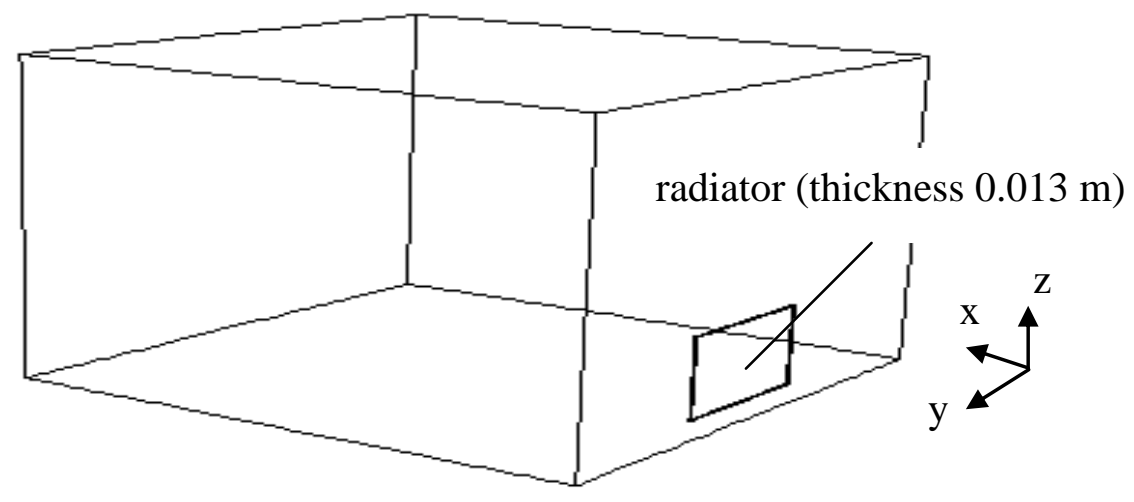

Figure 3. Computational domain geometry

The computational domain discretization is performed by means of finite volumes. The grid used is an unstructured mesh that contains tetrahedral elements. The principal demand that leads to an unstructured grid choice is given by the need of a better discretization in the regions with strong flow gradients. In fact, in the case of an unstructured mesh, this can be fulfilled by effortlessly refining only the zone where important flow variations occur, without adding unnecessary cells all over in the computational domain as normally happens in the structured grid approach. This important unstructured grid advantage has been used in our case to improve the plume area and walls boundary layers discretization.

On the other hand, there is no advantage to using a structured grid for our configurations in order to reduce the numerical diffusion since there is no relevant principal direction flow. It is known that the numerical diffusion appears within all numerical schemes. Besides, it is also established that the numerical diffusion is diminished when the flow is aligned with the mesh elements [17]. 
This is possible to achieve for simple flows (for instance the flow through a long duct) but it is not realizable for complex ones, as the flow can never be aligned with the grid.

Consequently, in order to properly discretisate the plume region, we first took into account a very fine discretization of the heat source. Therefore, based on a very fine surface mesh (quadrilateral elements) on the panel heater faces, we get a total of 112,000 hexahedral cells for the radiator discretization. This very fine discretization of the radiator faces also represents the support of an extremely fine 3D mesh for the fluid area that surrounds the heat source.

Unfortunately, the first simulations based on this discretization did not lead to acceptable results concerning the dynamic and thermal fields in the plume region. This is the reason why we defined an area in the vicinity of the heat source where we performed a mesh refinement. We present in Table 2 the extent of this region as well as the grid characteristics. The coordinates of the refinement mesh zone specified in Table 2 are related to the system of reference indicated in Figure 3. In fact, this area with higher density grid is located above the radiator along its entire length.

Table 2. Mesh refinement in the plume region

\begin{tabular}{cc}
\hline Refinement mesh zone (coordinates) & $\begin{array}{c}\text { Number of finite volumes } \\
\text { (before/after mesh refinement) }\end{array}$ \\
\hline $\mathrm{x}=0.001 / 0.12 \mathrm{~m} ; \mathrm{y}=1.4 / 2.6 \mathrm{~m} ; \mathrm{z}=0.8 / 2.5 \mathrm{~m}$ & $31,439 / 249,918$ \\
\hline
\end{tabular}

This technique has been successfully used by Markatos and Pericleous [18] for buoyancy driven cavities at high Rayleigh number $\left(\mathrm{Ra}=10^{16}\right)$. They progressively refined the mesh to reduce the numerical diffusion. In addition, the same approach has been employed in recent studies on related topics $[9,19]$.

Finally, the mesh for the entire computational domain discretization contains $1,417,653$ cells. It must be mentioned that this discretization has been used as a result of numerical mesh sensitivity tests undertaken in order to establish grid independent solutions.

\subsection{Turbulence modeling}

Our computations are based on a two-equation model, the realizable k- $\varepsilon$ model [20] that represents an adaptation of the standard k- $\varepsilon$ model. In fact, of the most common turbulence models (the classical models, based on Reynolds equations as the zero equation model - the mixing length model, the two equation model - k- $\varepsilon$ model and the second order closure models - 
the Reynolds stress equation model or those based on space filtered equations - large eddy simulation), the k- $\varepsilon$ model is still the most widely used and validated. On the other hand, in spite of its abundant successful computations for many industrially relevant flows, the k- $\varepsilon$ model has shown less accurate results for a specific range of flows (for instance, the spreading rate of axisymmetric jets is overpredicted or its precision is reduced while dealing with flows involving highly curved boundary layers). As a result, a number of revised versions of this two-equation model are proposed in the literature and one of them is the realizable form used in our study. Several studies [21-23] has revealed the superiority of the realizable k- $\varepsilon$ model in comparison with the classical k- $\varepsilon$ model for flows including boundary layers under strong adverse pressure gradients, separation or recirculation. These improvements are caused by a new formulation concerning the eddy viscosity and a new model dissipation rate equation too. We briefly present below these differences, mentioning that more complete elements about the realizable k- $\varepsilon$ model formulation are given in [20].

The eddy viscosity $\left(\mu_{t}\right)$ is determined from the same equation as in other k- $\varepsilon$ models:

$\mu_{t}=\rho C_{\mu} \frac{k^{2}}{\varepsilon}$

where $\rho$ represents the density; $\mathrm{k}$ and $\varepsilon$ correspond to the turbulence fields: turbulent kinetic energy and its rate of dissipation, respectively.

The difference is that the model coefficient $\mathrm{C}_{\mu}$ is no longer constant (usually 0.09) as in the standard model. Its value is a function of the mean strain $\left(S_{i j}\right)$ and rotation $\left(\Omega_{\mathrm{ij}}\right)$ rates, as well as of the turbulence parameters, $k$ and $\varepsilon$.

$$
C_{\mu}=\frac{1}{A_{0}+A_{S} \frac{U^{*} k}{\varepsilon}}
$$

with the model constants $A_{0}$ and $A_{S}$ obtained by: $A_{0}=4.04 ; A_{S}=\sqrt{6} \cos \Phi ; \Phi=\frac{1}{3} \arccos (\sqrt{6} W)$; $W=\frac{S_{i j} S_{j k} S_{k i}}{S} ; S=\sqrt{S_{i j} S_{i j}} ; U^{*}=\sqrt{S_{i j} S_{i j}+\Omega_{i j}^{*} \Omega_{i j}^{*}}$ and $\Omega_{i j}^{*}=\bar{\Omega}_{i j}-3 \varepsilon_{i j k} \omega_{k}, \bar{\Omega}_{i j}$ being the mean rate of rotation tensor in a rotating reference frame with the angular velocity $\omega_{\mathrm{k}}$. The Equation 2 makes reduce the overprediction of the eddy viscosity for flows with a high mean shear rate, fact that occurred in the case of the standard $\mathrm{k}-\varepsilon$ model. 
Further, the modeled transport equation for $\varepsilon$ is different from those used in the standard k- $\varepsilon$ model. In this case, this is based on the mean square vorticity fluctuation dynamic equation [20]:

$\overline{\mathrm{u}_{\mathrm{j}}} \frac{\partial \varepsilon}{\partial \mathrm{x}_{\mathrm{j}}}=\frac{\partial}{\partial \mathrm{x}_{\mathrm{j}}}\left[\left(v+\frac{v_{\mathrm{t}}}{\sigma_{\varepsilon}}\right) \frac{\partial \varepsilon}{\partial \mathrm{x}_{\mathrm{j}}}\right]+\mathrm{C}_{1} \mathrm{~S} \varepsilon-\mathrm{C}_{2} \frac{\varepsilon^{2}}{\mathrm{k}+\sqrt{v \varepsilon}}+\mathrm{C}_{1 \varepsilon} \frac{\varepsilon}{\mathrm{k}} \mathrm{C}_{3 \varepsilon} \mathrm{G}_{\mathrm{b}}$

In Equation 3, the left term represents the dissipation transport caused by the mean flow. Moreover, $v$ and $v_{t}$ stand for the cinematic viscosity and its eddy formulation respectively. Also, $\sigma_{\varepsilon}$ represents the turbulent Prandtl number for $\varepsilon(1.2)$ while $\mathrm{C}_{2}$ and $\mathrm{C}_{1 \varepsilon}$ are constants (1.9 and 1.44 correspondingly). In addition, the degree to which $\varepsilon$ is affected by the buoyancy is determined by means of constant $C_{3 \varepsilon}$. Finally, $G_{b}$ is the turbulent kinetic energy generation due to buoyancy, determined in a similar manner as in the classical k-E approach.

We notice that there is a new coefficient in Equation $3, \mathrm{C}_{1}$. Its value is obtained from:

$C_{1}=\max \left[0.43, \frac{\eta}{\eta+5}\right]$, where $\eta=\frac{S k}{\varepsilon}$

We observe also that the production term in the $\varepsilon$ equation - the second term on the right hand side of Equation 3, does not involve anymore the $k$ production as the other k- $\varepsilon$ models. It is supposed that this fact allows a more appropriate turbulence length scale.

On the other hand, regarding the modeled transport equation for $k$, this is exactly the same compared to the standard k- $\varepsilon$ model (including also the same approach for the turbulent kinetic energy source terms).

Finally, concerning the value of the turbulent Prandtl number which must be taken into account in our simulations, Kofoed and Popiolek [24] found out values between 0.3-0.8 based on literature review of studies dealing with thermal plumes. In fact, the turbulent Prandtl number does not have a constant value in all cross-sections of the plume along its axis. Moreover, Mierzwinski [25] show that the turbulent Prandtl number depends on the airflow structure: values below 0.5 for turbulent fields in plumes characterized by large dimensions vortical structures while values superior to 0.5 are recommended when small turbulent structures prevail in the airflow. In addition, Agator [26] show that in the case of a wall plume the turbulent length scale is strongly reduced and he used a value of 0.75 for the turbulent Prandtl number. This value lead to good agreement with experimental data on the affinity area of plume profiles. Consequently, 
taken into account the features of our study (wall plume), we adopt a larger value for the turbulent Prandtl number. Moreover, our computational domain is not limited to the plume's region, as a result we have to implement an appropriate value for the turbulent Prandtl number throughout the enclosure. Consequently, the value used in our simulations is 0.85 . We will see later that this value allowed us to correctly predict both the plume development and the thermoaeraulic field in the central area of the enclosure.

\subsection{Near wall airflow treatment}

There are three techniques usually employed in order to describe the near wall region:

- wall functions

- low Reynolds turbulence models

- two-layer zonal models

The first approach, based on the wall functions, has been very popular because it is economical and robust. This method allows important computational resource savings due to fait that the viscosity-affected near wall region is not resolved. Instead, semi-empirical formulas link the solution variables at the near wall cells and the analogous quantities on the wall. Unfortunately, the wall functions have originally been developed for forced convection flows, therefore their application is not suited for natural convection flows. There is significant evidence from the literature to demonstrate that the classic log-wall functions have been deficient at computing surface convection in room air flows in general, and in buoyancy driven flows in particular [27]. In existing two-equation "low Reynolds number" turbulence models, additional source/sink terms are included in the $k$ and $\varepsilon$ equations to account for viscous effects in regions with low turbulent intensity (next to the walls). A number of low Reynolds turbulence models are reviewed in [28]. Moreover, over the last 20 years modifications of "low Reynolds number" turbulence models mainly focused on the numerical formulation of the wall effects in the dissipation rate equation [29]. In this case, the computations impose a fin grid discretisation close to the walls since the viscosity-affected sublayer is this time solved. Nevertheless, these models were initially created for simple flows in order to determine the boundary layer velocity profiles next to isolated flat plates. Therefore, their application is not well matched for complex flows that include somehow complicated geometries and substantially different buoyancy induced flow regime caused by the presence of the heat source located within the enclosure, as happens in our study. 
Consequently, we finally use the two-layer zonal models to describe the near wall area in our computational domain since it is the only method that answers well at all our demands: strong body forces, boundary layer separations and relatively complicated geometries. This approach was employed with excellent results in [30] for modeling turbulent natural convection in enclosures with differentially heated vertical walls. In comparison with a classical low Reynolds number approach, this method allows an improved convergence, requires less mesh elements and introduces properly the length scale turbulence near wall distribution. In fact, the two-layer zonal model used comprises a separation of the computational domain into a viscosity-affected region and a fully turbulent region (where the realizable k- $\varepsilon$ model is applied). The border of these two zones is obtained by a wall distance based, turbulent Reynolds number:

$\operatorname{Re}_{y}=\frac{y \sqrt{k}}{v}$

where $y$ stands for the normal distance from the wall at the cell grid centres.

Furthermore, in the viscosity near-wall region $\left(\operatorname{Re}_{\mathrm{y}}<200\right)$, a one-equation turbulence model (only for the turbulent kinetic energy) is employed [31]. For this reason, the eddy viscosity and the dissipation rate of the turbulent kinetic energy are algebraically obtained by means of length scales:

$v_{\mathrm{t}}=\mathrm{C}_{\mu} \mathrm{L}_{\mu} \mathrm{k}^{\frac{1}{2}}$

$\varepsilon=\frac{\mathrm{k}^{\frac{3}{2}}}{\mathrm{~L}_{\varepsilon}}$

The length scales that appear in Eqs. (6) and (7) are based on the expressions [32]:

$$
\begin{aligned}
& \mathrm{L}_{\mu}=\mathrm{c}_{\mathrm{L}} \mathrm{y}\left[1-\exp \left(-\frac{\operatorname{Re}_{\mathrm{y}}}{\mathrm{A}_{\mu}}\right)\right] \\
& \mathrm{L}_{\varepsilon}=\mathrm{c}_{\mathrm{L}} \mathrm{y}\left[1-\exp \left(-\frac{\operatorname{Re}_{\mathrm{y}}}{\mathrm{A}_{\varepsilon}}\right)\right]
\end{aligned}
$$

where the constant values are given as follows: $\mathrm{c}_{\mathrm{L}}=\kappa_{\mathrm{C}} \mathrm{C}_{\mu}^{-\frac{3}{4}} ; \mathrm{A}_{\mu}=70$ and $\mathrm{A}_{\varepsilon}=2 \mathrm{c}_{\mathrm{L}}\left(\mathrm{C}_{\mu}=0.09\right.$ and $\kappa=0.42-$ Von Karman's constant). 


\subsection{Radiation modeling}

We have to introduce in our simulations the radiation heat transfers as the radiant heat fluxes between the computational domain surfaces become important compared to the heat transfer rate due to convection or conduction. The computations concerning the radiation heat transfers allow us to obtain proper wall surface temperatures as well as a correct heat source radiative rate heating power evaluation (see Table 8).

On the other hand, the radiative heat transfer modeling is based on the hypothesis that the air within the enclosure is a non-participating radiation medium despite of the fact that humid air contains water vapor which is an absorbing element in the infrared. This assumption has been used with satisfactory results in other similar studies [33]. In addition, all surfaces taken into account within the model (room walls and radiator faces) have the following properties: grey scattering surfaces (transmission and reflection).

In order to assess the radiative heat fluxes (multiple reflections that take place in the enclosure), we added at our principal equations governing the airflow the discrete ordinates (DO) radiation model, more precisely the conservative DO version, the finite-volume method [34]. It must be mentioned that the same approach have been successfully used in other analogous studies $[11,12]$. This method resolves the radiative transfer equation for a finite number of discrete solid angles (the number of radiation intensity transport equation solved is given by the amount of discrete solid angles defined). As a result, the conservative DO variant, based on the finitevolume method converts this equation in a radiation intensity transport equation, numerically solved as the airflow model equations.

The solid angles for the DO radiation model employed are obtained by means of a fixed vector direction in the spatial coordinates $(\mathrm{x}, \mathrm{y}, \mathrm{z})$. In fact, the angular discretization is based on control angles that determine the discretization of each octant in the angular space. In our case, we employed a minimum angular discretization for the implementation of the DO method: two control angles for the polar and azimuthal angles to locate the vector direction in space. Based on the results obtained, this relatively roughly angular discretization is sufficient to correct evaluate the radiative heat transfers. This quite poor but satisfactory angular discretization must be correlated with the simplicity of our geometry that allows somewhat simplified radiation calculation. 
Finally, there were another alternatives to compute the radiation heat transfer between the surfaces of the computational domain (enclosure walls and heat source faces): the Discrete Transfer Radiation Model (DTRM) - employed in several similar researches [9,19,23,35] or P-1 radiation model (the simplest case of the more general $\mathrm{P}-\mathrm{N}$ radiation model). However, the DTRM successful application demands a large number of rays and this implies more intensive central processing unit (CPU) computations. On the contrary, in the case of the DO radiation model, accurate results can be achieved with reasonable computational cost. Concerning the P-1 model, this approach typically overestimates the radiation heat fluxes for simulations involving concentrated heat sources [36]. Consequently, the DO method is the most appropriate radiation model for this kind of applications.

\subsection{Thermal boundary conditions}

Concerning the boundary conditions, we make use of the measurements. As a result, we impose a convective heat transfer to the walls, providing the external convective heat transfer coefficient $\left(\mathrm{h}_{\mathrm{ce}}\right)$ and the external temperature measured in the adjacent test room thermal guard area $\left(\mathrm{T}_{\mathrm{e}}\right)$ in order to determine the heat flux to the wall $(\mathrm{q})$ :

$$
\mathrm{q}=\mathrm{h}_{\mathrm{ci}}\left(\mathrm{T}_{\mathrm{i}}-\mathrm{T}_{\mathrm{w} 1}\right)+\mathrm{q}_{\mathrm{r}}=\lambda \frac{\mathrm{T}_{\mathrm{w} 1}-\mathrm{T}_{\mathrm{w} 2}}{\delta}=\mathrm{h}_{\mathrm{ce}}\left(\mathrm{T}_{\mathrm{w} 2}-\mathrm{T}_{\mathrm{e}}\right)
$$

where $\mathrm{h}_{\mathrm{ci}}$ represents the internal local convection heat transfer coefficient based on the local flow field conditions (temperature, velocity as well as turbulence level); $\mathrm{T}_{\mathrm{w} 1}$ and $\mathrm{T}_{\mathrm{w} 2}$ are wall inner and outer surface temperatures. In the same time, $\mathrm{T}_{\mathrm{i}}$ corresponds to the local fluid (air) temperature and $\mathrm{q}_{\mathrm{r}}$ stands for the radiative heat flux to the internal surfaces of the enclosure walls (based on the discrete ordinates radiation model as described above and taking into account the emissivity of the walls inner surface using experimental results - see section 2). Finally, $\lambda$ and $\delta$ are the wall thermal conductivity and thickness, respectively (their values are based on experimental data - see also section 2).

It is worthwhile to mention that the thermal boundary conditions imposed by Eq. (10) represent the only alternative to properly take into account the convection-radiation coupling, the internal surface temperature being in this case the result of these two modes of heat transfer to the wall. On the other hand, in the literature [37], the correlations given to determine the external heat transfer coefficients lead unfortunately to an extended interval. The values vary from 6 to 17.1 
$\mathrm{W} / \mathrm{m}^{2} \mathrm{~K}$ for an air velocity of $1 \mathrm{~m} / \mathrm{s}$ as is the case inside the adjacent test room thermal guard area. Therefore, we had to carry out numerical manipulations in order to find out the better value to enforce. Finally, the value that allows a correct plume development is $12 \mathrm{~W} / \mathrm{m}^{2} \mathrm{~K}$, somehow in the middle of the mentioned interval.

\subsection{Heat source modeling}

It is worthwhile to note that there are several potential strategies to deal with the CFD heat source integration. For instance, we can mention here the approaches within the International Energy Agency, Annex 20 - 1991 for the test case D - "free convection with radiator" reviewed by Beghein [15]: radiator boundary conditions introduced as uniform heat fluxes at heater faces (regrettably, such numerical approaches, based also on standard k- $\varepsilon$ turbulence model and wall functions, have not given acceptable results as the resulting radiator surface temperatures are overestimated comparing with the measurements); simplified approach, the heater is replaced by a convective heat source that does not represent an obstacle for the airflow (nevertheless, in our case, we can not neglect the radiative heat transfers as the experimental results show that more than $50 \%$ of the total radiator heat power takes place by radiation). The same method, using fixed thermal flux on the radiator surface, is taken into account in other recent studies [12]. Unfortunately, such a method is not adequate in our case since the radiator thickness is very reduced compared to its length and height. In addition, this approach leads to "a priori" imposition of heat exchanges while these deeply depend on the flow characteristics near the heat source. Other possible methods are based on fixed temperature set for the whole radiator surface $[19,38,39]$. Despite the good results that follow from this methodology, there is a lack of practical application because the heat source surface temperatures must be known. Moreover, these temperatures are not usually accessible for ordinary situations where there are not detailed experimental data. On the other hand, this approach "freezes" the surface temperature although these depend on local flow conditions in the vicinity of the heat source.

Consequently, the radiator integration in our numerical model is performed by adding a term

source in the energy balance equations of the cells representing the radiator discretization. More precisely, it is a volumetric heat generation rate $\left(\mathrm{S}_{\mathrm{h}}\right)$ accordingly to the source power $(\mathrm{Q})$ and its dimensions ( $\mathrm{V}$ - volume) which is uniformly distributed throughout the radiator: 
$\mathrm{S}_{\mathrm{h}}=\frac{\mathrm{Q}}{\mathrm{V}}$

As a result, we have the following equation (solid medium with internal heat source, Poisson's equation), assuming homogenous and isotropic medium with a thermal conductivity $\lambda$ independent of temperature:

$\frac{\partial}{\partial \mathrm{x}_{\mathrm{i}}}\left(\lambda \frac{\partial \mathrm{T}}{\partial \mathrm{x}_{\mathrm{i}}}\right)+\mathrm{S}_{\mathrm{h}}=0$

The accuracy of this approach is demonstrated by the results obtained in term of test room thermal balance as well as those related to the plume dynamic and thermal fields over the radiator (see the next section regarding the results achieved). We present here the experimental numerical comparisons in term of radiator surface temperatures (Table 3). These temperatures are attained by means of heat transfer computations: conduction in solid (radiator) cells, convection in fluid cells in the surrounding area of the heat emmiter and radiation on the heat source surfaces. It can be noticed that the numerical values are extremely close to experimental data (difference less than $2 \%$ ).

Table 3. Average radiator surface temperature $\left({ }^{\circ} \mathrm{C}\right)$

\begin{tabular}{ccc}
\hline Test & Experimental [14] & Numerical \\
\hline R1 & 73.4 & 72.1 \\
R2 & 73.0 & 71.6 \\
\hline
\end{tabular}

Finally, we mention that another two techniques were tested during our simulations but their results were relatively disappointing. Firstly, we divided the heater into two distinct regions of same height but with different heat power repartition in each of them (see Figure 4.A). As a result, we noticed only slight temperature improvements behind the heater. Moreover, these improvements simply occurred very close to the radiator, beyond this, the thermal field being over predicted.

The second method was based on a linear heat power variation on the radiator height (Figure 4.B) but we did not see any significant results improvements comparing with the basic approach (volumetric heat generation rate) previously introduced.

Consequently, taken into account its simplicity, we still prefer the approach based on the uniform volumetric heat distribution. Incidentally, it is worthwhile to emphasize the main 
advantages of this method (beyond its precision): straightforward to accomplish and to extrapolate, no need of measurements (it is enough to provide the heat source power and dimensions), no alteration of flow conditions near the heat emmiter.

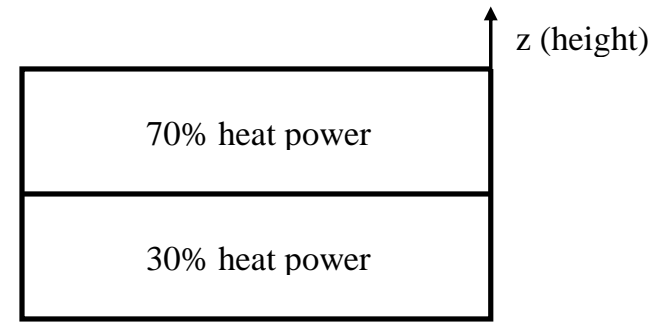

A

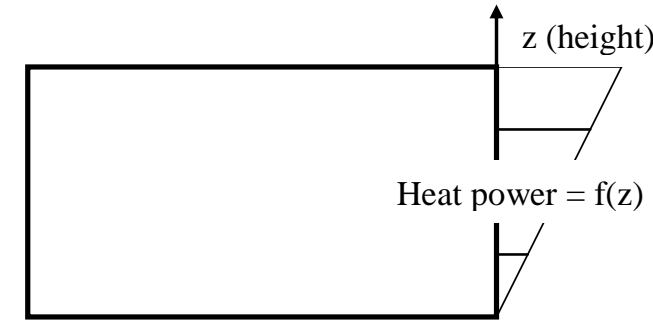

B

Figure 4. Other proposed heat source integration approaches

\section{Results}

Since the main objective of this study is to assess the capacity and the precision of our CFD model to predict the behavior of the thermal coupling between the room and the radiator, we present below experimental - numerical comparisons with regard to the heat transfer to the walls, heat source behavior and plume characteristics.

\subsection{Heat transfer to walls}

First of all, we present in the Table 4 the numerical results concerning the test room overall balance. We see that there is a good agreement between the heat generation inside the room and its heat losses, meaning that the enclosure heat balance is scrupulously respected (the differences do not exceed $0.4 \%$ ). This expresses the necessary condition to support the accuracy of the numerical model both in terms of the convergence and numerical results concerning heat transfer in the enclosure.

Table 4. Enclosure thermal balance (numerical results)

\begin{tabular}{cccc}
\hline Test & Radiator heat power $(\mathrm{W})$ & Overall room heat balance $(\mathrm{W})$ & Error $(\%)$ \\
\hline R1 & 900 & 896.8 & 0.35 \\
R2 & 881 & 878.5 & 0.28 \\
\hline
\end{tabular}

Moreover, Table 5 shows the total heat rate through the cooled walls of the enclosure during the tests. Taking into consideration the measurements precision, we consider the results satisfactory 
(the maximum difference is $13.3 \%$ - ceiling, test R2). These results confirm the pertinence of the two-layer approach employed to describe the air flow and the surface convection in buoyancy driven flows within enclosures.

Table 5. Heat flux to the "cooled" walls (W)

\begin{tabular}{cccc}
\hline Test & Wall & Experimental [14] & Numerical \\
\hline \multirow{2}{*}{ R1 } & South (wall behind the heater) & 346.7 & 374.8 \\
& North (heater opposite wall) & 252.0 & 239.2 \\
\hline \multirow{2}{*}{ R2 } & South (wall behind the heater) & 316.3 & 352.4 \\
& Ceiling & 243.2 & 275.5 \\
\hline
\end{tabular}

In line with this, we present also direct experimental - numerical confrontations regarding the internal convective heat transfer coefficient at the ceiling (Table 6). Unfortunately, there are no experimental values for other convective heat transfer coefficients of the enclosure walls.

Table 6. Convective heat transfer coefficients (averaged value) at ceiling $\left(\mathrm{W} / \mathrm{m}^{2} \mathrm{~K}\right)$

\begin{tabular}{cccc}
\hline Test & Experimental [14] & Numerical & Error $(\%)$ \\
\hline R1 & 4.9 & 5.3 & 8.2 \\
R2 & 7.5 & 8.4 & 12.0 \\
\hline
\end{tabular}

Nevertheless, we compared in Table 7 our numerical results concerning convection heat transfer at internal surfaces of the enclosure with data available in the literature. We tried to take into account only the results based on the same configurations (experimental cell heated by radiator). In addition, we added values from correlations dealing with free convection heat transfer at walls. These correlations take into account the temperature difference wall - indoor air by the means of three coefficients $\mathrm{a}, \mathrm{b}$ and $\mathrm{n}$ in the following way:

$\mathrm{h}_{\mathrm{c}}=\mathrm{a}\left(\mathrm{T}_{\mathrm{w}}-\mathrm{T}_{\mathrm{a}}\right)^{\mathrm{n}}+\mathrm{b}$

where $h_{c}-$ convective heat transfer coefficient, $T_{w}-$ wall surface temperature and $T_{a}-$ air temperature.

It is worthwhile to mention that the coefficients involved in the Eq. (13) are generally obtained from studies of natural convection for horizontal or vertical plates.

\subsection{Heat source behavior}

Table 8 shows experimental - numerical comparisons in term of radiative and convective heating power in function of the total radiator heating capacity. Based on the results obtained, we remark 
a good radiator behaviour prediction (the maximum difference is $8.8 \%$ - convective power, test R1).

Table 7. Convective heat transfer coefficients (averaged value) at vertical walls $\left(\mathrm{W} / \mathrm{m}^{2} \mathrm{~K}\right)$

\begin{tabular}{cccc}
\hline Test & Wall & Experimental* / Correlation** & Numerical \\
\hline \multirow{4}{*}{ R1 } & South (wall behind the heater) & 5.2 Lebrun [14]* & 5.5 \\
& North (heater opposite cool wall) & $3 \div 4.5$ Ngendakumana [40]* & 3.4 \\
& East & $1.1 \div 2.9[41]^{* *}$ & 1.1 \\
& West & $1.1 \div 2.9[41]^{* *}$ & 0.8 \\
\hline \multirow{4}{*}{ R2 } & South (wall behind the heater) & 5.2 Lebrun [14]* & 6.4 \\
& North (heater opposite wall) & $3 \div 4.5$ Ngendakumana [40]* & 1.9 \\
& East & $1.1 \div 2.9[41]^{* *}$ & 1.6 \\
& West & $1.1 \div 2.9[41]^{* *}$ & 1.6 \\
\hline
\end{tabular}

The heater surface temperatures confirm this: during the R2 configuration, the heater surface temperature revealed is $73.0^{\circ} \mathrm{C}$ [14] while the maximum radiator face temperature numerically obtained is $71.85^{\circ} \mathrm{C}$ (see also Table 3).

Table 8. Radiative and convective heating power of the heat source (W)

\begin{tabular}{lccc}
\hline Test & Radiator heating power & Experimental & Numerical \\
\hline \multirow{2}{*}{ R1 } & Radiative & 522 & 491.4 \\
& Convective & 375.3 & 408.6 \\
\hline \multirow{2}{*}{ R2 } & Radiative & 519.8 & 482.8 \\
& Convective & 372.9 & 398.2 \\
\hline
\end{tabular}

On the other hand, the good numerical representation of the radiator convective emission is also supported by comparisons with empirical correlations. Consequently, we present in Table 9 the values obtained in terms of Nusselt number, $\mathrm{Nu}$ in order to characterize the natural convection around the heat source. The numerical results are compared to data acquired from Churchill and Chu experimental correlations [18] expressing the heat transfer for vertical plates in natural convection:

$$
\mathrm{Nu}=\left\{0,825+\frac{0,387 \mathrm{Ra} a^{\frac{1}{6}}}{\left[1+\left(\frac{0,492}{\mathrm{Pr}}\right)^{\frac{9}{16}}\right]^{\frac{8}{27}}}\right\}^{2}
$$


In Eq. (14) the Rayleigh number, $\mathrm{Ra}$ is based on the radiator height as characteristic length and the reference temperature difference takes into account the temperature difference between the radiator surface temperature and the air temperature in the middle of the enclosure. The Prandtl number, Pr is related to the air (0.7).

Table 9. Nusselt number around the radiator

\begin{tabular}{cccc}
\hline Test & Churchill and Chu [18] & Numerical & Error $(\%)$ \\
\hline R1 & 220.8 & 238.0 & 7.8 \\
R2 & 220.9 & 231.7 & 4.9 \\
\hline
\end{tabular}

The results of Table 9 put in evidence the overestimation of radiator convective heat transfer coefficients. This explains the slight overestimation of the convective fraction of the heater revealed in the numerical model.

\subsection{Radiator plume analyze}

Thermal plumes (even weak ones) are essential factors causing air movement in heated rooms without ventilation systems. Therefore, it is required a good knowledge of their developing vertical velocity and temperature excess distributions in order to predict the air quality and occupant comfort level in the enclosures.

For this reason, we carried out detailed experimental - numerical comparisons at a succession of elevations over the heater (in the room median vertical plane) in term of mean air velocity and temperature. We exemplify these comparisons by the values obtained at two heights, $1.51 \mathrm{~m}$ and $1.96 \mathrm{~m}$, meaning $0.71 \mathrm{~m}$ and $1.16 \mathrm{~m}$ over the radiator.

It is worthwhile to note that the comments presented below, related to the experimental numerical plume characteristics comparisons, are valid for all the elevations taken into account but not presented here.

We notice (Figure 5, test R1) that the velocity distribution is well represented, (particularly near to the radiator) the maximal experimental and numerical values for each height being extremely closed: at $\mathrm{z}=1.51 \mathrm{~m}, 0.55 \mathrm{~m} / \mathrm{s}$ (experimental) opposed to $0.53 \mathrm{~m} / \mathrm{s}$ (numerical) meaning a error of $3.6 \%$ and at $\mathrm{z}=1.96 \mathrm{~m}$, an experimental value of $0.6 \mathrm{~m} / \mathrm{s}$ versus a numerical one of $0.57 \mathrm{~m} / \mathrm{s}$, the error being in this case 5\%. On the other hand, these maximal values are also identified practically at the same $\mathrm{x}$ coordinates. In fact, in the radiator neighborhood, the dynamic field is 
accurately taken into account in the numerical model. More exactly, at $\mathrm{z}=1.51 \mathrm{~m}$ and for $\mathrm{x}$ between 0 and $0.2 \mathrm{~m}$, the error varies only between 0.5 and $15 \%$ and at $\mathrm{z}=1.96 \mathrm{~m}(\mathrm{x}=0-0.25$ $\mathrm{m}$ ), the differences are limited between 2 and 15\%. On the contrary, away from these limits, the velocities are overestimated. This means that the momentum spread is more important in the numerical model, probably due to the turbulence model, which is still too diffusive.

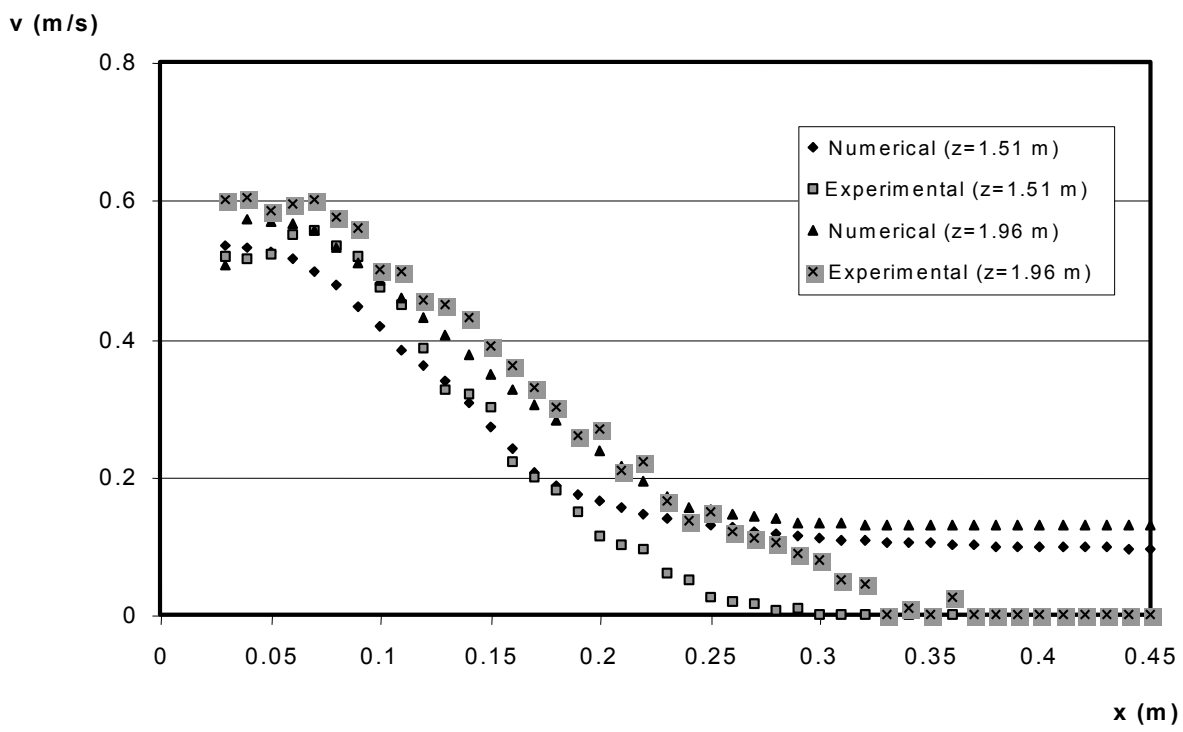

Figure 5. Velocity profiles over the radiator, test R1

The temperature excess distributions are presented in the Figure 6 for the test R1. We remark a slightly numerical temperature overprediction. However, the overall accord between the measurements and simulations is more than reasonable.

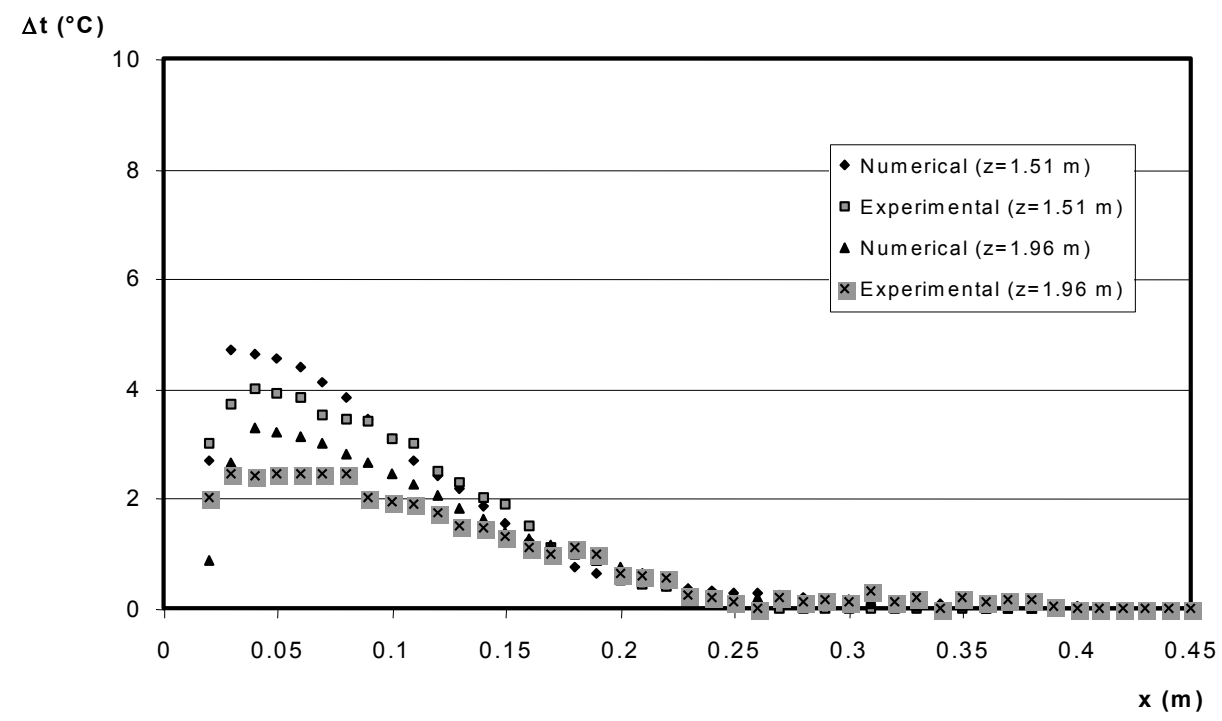


Figure 6. Temperature profiles over the radiator, test R1

The same velocity distributions are illustrated in the Figure 7 for the configuration R2. We notice similar trends as in the previous analyze concerning the test $\mathrm{R} 1$. The comparisons regarding the maximal values show however that the cold ceiling influence is more difficult to take into consideration within the numerical model since there is a more important difference at $\mathrm{z}=1.96$ $\mathrm{m}, 11.5 \%(0.61 \mathrm{~m} / \mathrm{s}-$ experimental and $0.54 \mathrm{~m} / \mathrm{s}-$ numerical $)$. On the contrary, at $\mathrm{z}=1.51 \mathrm{~m}$, the maximal value is almost perfectly reached: $0.53 \mathrm{~m} / \mathrm{s}$ - measured value comparing with a numerical one of $0.52 \mathrm{~m} / \mathrm{s}(1.8 \%)$.

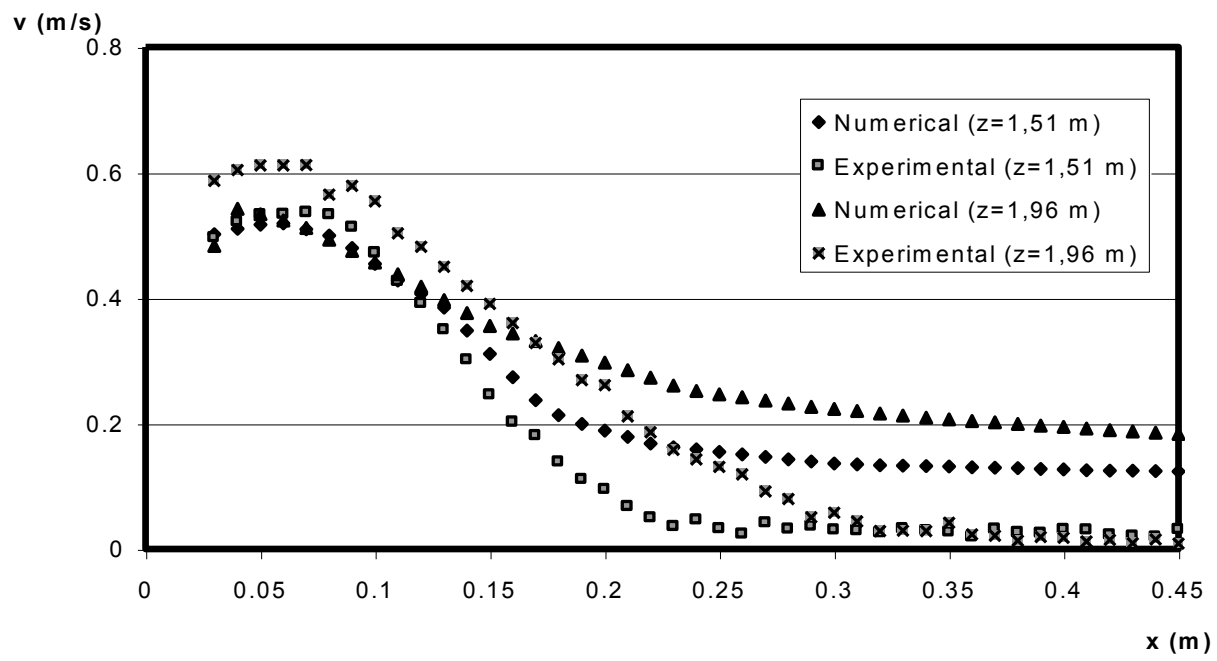

Figure 7. Velocity profiles over the radiator, test R2

Finally, the temperature profiles for the configuration R2 are reported in the Figure 8. Once more, the computations lead to satisfactory results, compared to experimentation. 


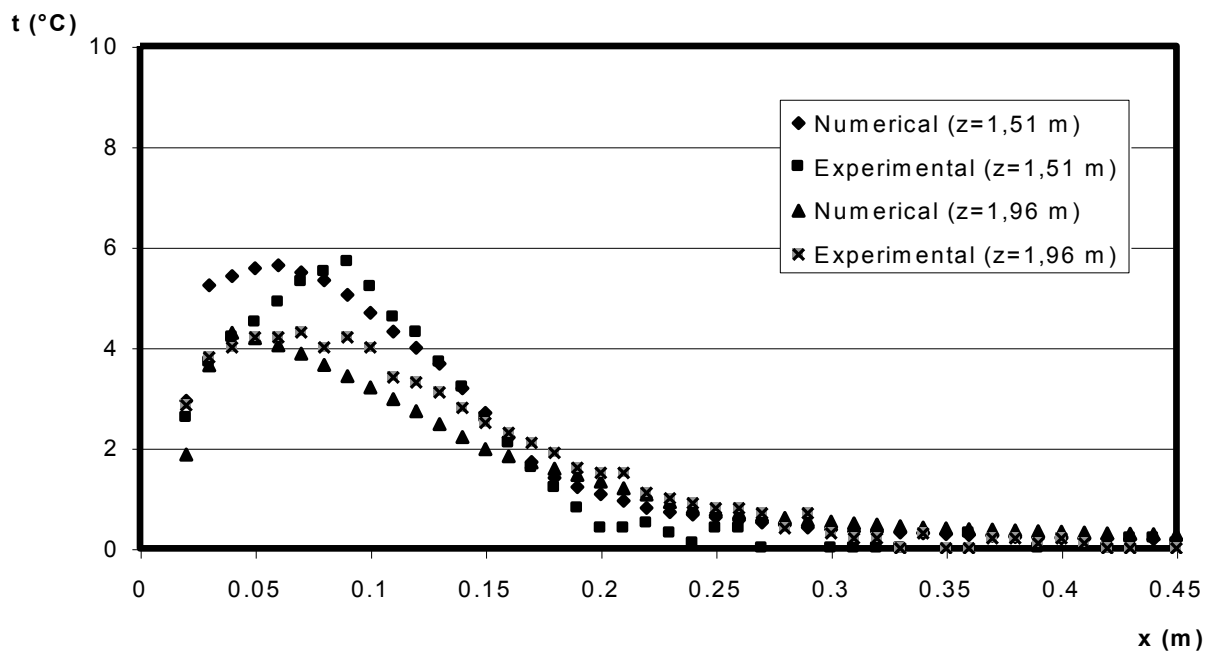

Figure 8. Temperature profiles over the radiator, test R2

\section{Conclusions}

The model which has been presented leads to realistic description of heat transfer through walls as well as to correct heat source behavior and plume expansion representation. As a result, such a methodology can be applied in works dealing with internal natural convective flows. Moreover, based on the quite accurate results achieved, this approach could be applied to correct assess the convective heat transfer coefficients in buoyancy driven flows within enclosures, knowing that there is a obvious need for precise estimations of this parameter, its value being usually related in the literature to isolated flat plates. However, before doing this, thoroughly studies ought to be carried out. Furthermore, this works should be correlated with more complete and precise experimentations (as infrared measurements). In addition, the external thermal boundary conditions (convective exchanges) must be also reconsidered to determine the exact computation sensitivity to this.

On the other hand, the correct plume representation allows us to obtain reliable values concerning the heat flow as well as the air induction in the plume. This generates an accurate airflow prediction within the heated room. Moreover, the simplified heat source approach can be used for other localized source of heat at floor level.

\section{Acknowledgement}


Thanks are due to Professor Christian Inard (University of La Rochelle, France) for his cooperation concerning the experimental data.

\section{References}

[1] F. Song, B. Zhao, X. Yang, Y. Jiang, V. Gopal, et al., A new approach on zonal modeling of indoor environment with mechanical ventilation, Building and Environment 43 (2008) 278-286

[2] A.C. Megri, F. Haghighat, Zonal Modeling for Simulating Indoor Environment of Buildings: Review Recent Developments and Applications, HVAC\&R Research 13 (2007) 887-905

[3] B. Griffith, Q.Y. Chen, Framework for Coupling Room Air Models to Heat Balance Model Load and Energy Calculations, HVAC\&R Research 10 (2004) 91-111

[4] S.H. Ho, L. Rosario, M.M. Rahman, Three-dimensional analysis for hospital operating room thermal comfort and contaminant removal, Applied Thermal Engineering 29 (2009) 2080-2092

[5] M. Aghakhani, G. Eslami, Thermal comfort Assessment of Underfloor vs. Overhead Air Distribution System, Journal of Applied Sciences 12 (2012) 473-479

[6] G. Gan, Simulation of buoyancy-driven natural ventilation of buildings - Impact of computational domain, Energy and Buildings 42 (2010) 1290-1300

[7] T. Kim, S. Kato, S. Murakami, Indoor cooling/heating load analysis based on coupled simulation of convection, radiation and HVAC control, Building and Environment 36 (2001) 901-908

[8] T. Catalina, J. Virgone, F. Kuznik, Evaluation of thermal comfort using combined CFD and experimentation study in a test room equipped with a cooling ceiling, Building and Environment 44 (2009) 1740-1750

[9] M.D. Ahanj, M. Rahimi, A.A. Alsairafi, CFD modeling of a radiant tube heater, International Communications in Heat and Mass Transfer 39 (2012) 432-438

[10] F. Moukalled, S. Verma, M. Darwish, The use of CFD for predicting and optimizing the performance of air conditioning equipment, International Journal of Heat and Mass Transfer 54 (2011) 549-563

[11] R. Gao, A. Li, O. Zhang, H. Zhang, Comparison of indoor air temperatures of different under-floor heating pipe layouts, Energy Conversion and Management 52 (2011) 1295-1304

[12] A. Ploskic, S. Holmberg, Heat emission from thermal skirting boards, Building and Environment 45 (2010) 1123-1133

[13] A. Sreshthaputra, J. Haberl, M.J. Andrews, Improving building design and operation of a Thai Buddhist temple, Energy and Buildings 36 (2004) 481-494

[14] C. Inard, Contribution to the study of the thermal coupling of a heat emitter with a room (in French), PhD. Thesis, INSA Lyon, France, 1988 
[15] C. Beghein, Contribution to the numerical study of thermosolutal natural convection in a cavity. Application of pollutant diffusion in rooms (in French), PhD. Thesis, INSA Lyon, France, 1992

[16] V.I. Polezhaev, M.N. Myakshina, S.A. Niktin, Heat transfer due to buoyancy-driven convective interaction in enclosures: Fundamentals and applications, International Journal of Heat and Mass Transfer 55 (2012) 156-165

[17] B.E. Launder, W.C. Reynolds, W. Rodi, J. Mathieu, D. Jeandel, Turbulence models and their applications, Eyrolles, Paris, 1984.

[18] S. Kakaç, R.K. Shah, W. Aung, Handbook of Single-Phase Convective Heat Transfer, John Wiley \& Sons, New York, 1987.

[19] A.K.A. Shati, S.G. Blakey, S.B.M. Beck, The effect of surface roughness and emissivity on radiator output, Energy and Buildings 43 (2011) 400-406

[20] T.H. Shih, W.W. Liou, A. Shabbir, Z. Yang, J. Zhu, A new k- $\varepsilon$ eddy viscosity model for high Reynolds number turbulent flows, Computers Fluids 24 (1995) 227-238

[21] C. Teodosiu, G. Rusaouen, S. Laporthe, Improvement of CFD application in ventilated enclosures a test case, Proceedings of $7^{\text {th }}$ International Conference on Air Distribution in Rooms ROOMVENT, 2000, Reading, UK.

[22] C. Teodosiu, G. Rusaouen, R. Hohotă, Influence of boundary conditions uncertainties on the simulation of ventilated enclosures, Numerical Heat Transfer, Part A: Applications - An International Journal of Computation and Methodology 44 (2003) 483-504

[23] S. Hussain, P.H. Oosthuizen, Validation of numerical modeling of conditions in an atrium space with a hybrid ventilation system, Building and Environment 52 (2012) 152-161

[24] P. Kofoed, Z. Popiolek, Turbulent Diffusion of Heat and Momentum in Buoyant Plumes, Proceedings of $4^{\text {th }}$ International Conference on Air Distribution in Rooms - ROOMVENT, 1994, Krakow, Poland.

[25] S. Mierzwinski, Testing and Modelling of Thermal Plumes in Rooms, Proceedings of International Symposium on Room Air Convection and Ventilation Effectiveness - ISRACVE, 1992, Tokyo, Japan.

[26] J.M. Agator, Contribution to the turbulent structure study of a thermal plume with axial symmetry. Plume interaction with its environment limit (in French), PhD. Thesis, University of Poitiers, France, 1983.

[27] I. Beausoleil-Morrison, The adaptive coupling of heat and air flow modelling within dynamic whole-building simulation, PhD. Thesis, University of Strathclyde, Glasgow, UK, 2000

[28] V.C. Patel, W. Rodi, G. Scheuerer, Turbulence models for near-wall and low Reynolds number flows - a review, AIAA Journal 23 (1985) 1308-1319 
[29] F. Billard, D. Laurence, A robust $\mathrm{k}-\varepsilon-\mathrm{v}^{2} / \mathrm{k}$ elliptic blending turbulence model applied to near-wall, separated and buoyant flows, International Journal of Heat and Fluid Flow 33 (2012) 45-58

[30] K.J. Hsieh, F.S. Lien, Numerical modeling of buoyancy-driven turbulent flows in enclosures, International Journal of Heat and Fluid Flow 25 (2004) 659-670

[31] M. Wolfstein, The velocity and temperature distribution of one-dimensional flow with turbulence augmentation and pressure gradient, International Journal of Heat and Mass Transfer 12 (1969) 301-318

[32] H.C. Chen, V.C. Patel, Near-wall turbulence models for complex flows including separation, AIAA Journal 26 (1988) 641-648

[33] M. Tabarki, S. B. Mabrouk, The coupling in transient regime between the modelings of thermal and mass transfers inside a heated room and its radiator, Heat Mass Transfer 48 (2012)1889-1901

[34] R. Siegel, J.R. Howell, Thermal radiation heat transfer, Taylor \& Francis, Washington DC, 1992.

[35] S. Hussain, P.H. Oosthuizen, Numerical investigations of buoyancy-driven natural ventilation in a simple atrium building and its effect on the thermal comfort conditions, Applied Thermal Engineering 40 (2012) 358-372

[36] S.S. Sazhin, E.M. Sazhina, O. Faltsi-Saravelou, P. Wild, The P-1 model for thermal radiation transfer: advantages and limitations, Fuel 75 (1996) 289-294

[37] A. Roldan, Thermo-aeraulic study of building envelopes (in French), PhD. Thesis, INSA Lyon, France, 1985

[38] J. Are Myhren, S. Holmberg, Design considerations with ventilation-radiators: Comparisons to traditional two-panel radiatos, Energy and Buildings 41 (2009) 92-100

[39] G. Sevilgen, M. Kilic, Numerical analysis of air flow, heat transfer, moisture transport and thermal comfort in a room heated by two-panel radiators, Energy and Buildings 43 (2011) 137-146

[40] H. Bouia, Simplified modeling of mixed internal convection air flow: application to thermalaeraulic transfers in enclosures (in French), PhD. Thesis, University of Poitiers, France, 1993

[41] C. Teodosiu, Modeling of technical systems in the field of building equipment using CFD codes (in French), PhD. Thesis, INSA Lyon, France, 2001 